\title{
Regularity of Solutions to the Quaternionic Monge-Ampère Equation
}

\author{
Sławomir Kołodziej ${ }^{1} \cdot$ Marcin Sroka $^{1}$
}

Received: 31 May 2019 / Published online: 26 March 2020

(c) The Author(s) 2020

\begin{abstract}
The regularity of solutions to the Dirichlet problem for the quaternionic MongeAmpère equation is discussed. We prove that the solution to the Dirichlet problem is Hölder continuous under some conditions on the boundary values and the quaternionic Monge-Ampère density from $L^{p}(\Omega)$ for $p>2$. As a step towards the proof, we provide a refined version of stability for the weak solutions to this equation.
\end{abstract}

Keywords Monge-Ampere equation · Pluripotential theory $\cdot$ Subharmonic functions

Mathematics Subject Classification Primary: 32U05 · Secondary: 35D30

\section{Introduction}

The theory of quaternionic plurisubharmonic functions in $\mathbb{H}^{n}$ was initiated by Alesker in [1]. He points out there that actually the definition of quaternionic plurisubharmonic function was suggested by Henkin. In particular, the quaternionic Monge-Ampère operator was defined in [1] for continuous functions. In the case of smooth functions, this is the Moore determinant, cf. [17], of the quaternionic Hessian. This will be explained in details in Preliminaries. In [2], the Dirichlet problem for this operator was solved for the right-hand side continuous up to the boundary and a continuous boundary data. It involved the methods based on the approach of Bedford and Taylor [6,7]. Later, the theory was further generalized to the case of hypercomplex manifolds by Alesker

Dedicated to the memory of Professor Gennadi Henkin.

$凶 \quad$ Sławomir Kołodziej

Slawomir.Kolodziej@im.uj.edu.pl

Marcin Sroka

Marcin.sroka@im.uj.edu.pl

1 Faculty of Mathematics and Computer Science of Jagiellonian University, Łojasiewicza 6, 30-348 Kraków, Poland 
and Verbitsky, cf. [4], and resulted in posting the, still unsolved, quaternionic Calabi Conjecture on HKT-manifolds, cf. [5]. Recently, the Bedford and Taylor theory was adopted to the quaternionic setting by Wan and Wang in [23]. This was achieved by introducing the formal operators $d_{0}, d_{1}$ in $\mathbb{H}^{n}$ being analogues, in many ways, of to $\partial, \bar{\partial}$ from $\mathbb{C}^{n}$. This approach was continued in $[22,24]$ and allowed to obtain many results known from the plurisubharmonic setting, most notably the comparison principle which is the main tool of the pluripotential theory.

On $\mathbb{H}^{n}$, one naturally defines differential operators $\partial$ and $\partial_{J}$ appearing for example in the quaternionic Dolbeault complex on any hypercomplex manifold, cf. [21]. For this reason, the second-named author studied in [20] the relation between $d_{0}, d_{1}$ and $\partial, \partial_{J}$ showing that they agree and thus the theory developed by Alesker is compatible with the one introduced in [23]. Another result of [20] is the solvability of the Dirichlet problem for densities from $L^{p}$ for any $p>2$ and a continuous boundary condition. Furthermore, this bound on the exponent was shown to be optimal and thus constitutes the analogue of first author's result on the complex Monge-Ampère equation, cf. $[14,16]$. In the meantime, Zhu has shown in [25] that when the initial data, i.e. a density and the boundary condition are smooth the solution is smooth as well. The methods of that paper are based on the classical paper by Caffarelli et al. [8].

Let us also mention that an alternative direction of generalizing the pluripotential theory was developed by Harvey and Lawson in [12,13] for calibrated manifolds. In the case of $\mathbb{H}^{n}$, their approach agrees with the one described above, cf. [3].

In this note, we show Hölder regularity for the weak solutions obtained in [20]. This is the content of Theorem 3 below, stating that when the boundary condition is in $C^{1,1}(\partial \Omega)$ and the quaternionic Monge-Ampère density is bounded near the boundary then the solution is $\alpha$-Hölder continuous and a bound on $\alpha$ is provided. The method of the proof is based on the one presented in [11] for the complex Monge-Ampère equation and further developed in [18] for the complex Hessian equation. It requires the refined version of a stability estimate, cf. Proposition 4 in [20], which we prove in Sect. 3. The proof of the regularity theorem is presented in Sect. 4. All the necessary facts from quaternionic pluripotential theory are presented in the section below.

\section{Preliminaries}

We treat $\mathbb{H}^{n}=\left\{\left(q_{0}, \ldots, q_{n-1}\right) \mid q_{i} \in \mathbb{H}\right\}$ as a right $\mathbb{H}$ module, where $\mathbb{H}=\left\{x_{0}+\right.$ $\left.x_{1} \mathfrak{i}+x_{2} \mathfrak{j}+x_{3} \mathfrak{k} \mid x_{i} \in \mathbb{R}\right\}$ and $\mathfrak{i}^{2}=\mathfrak{j}^{2}=\mathfrak{k}^{2}=\mathfrak{i j k}=-1$. We often use the following identifications

$$
\begin{aligned}
& \mathbb{H}^{n} \ni\left(z_{2 i}+\mathfrak{j} z_{2 i+1}\right)_{i=0}^{n-1} \longmapsto\left(z_{j}\right)_{j=0}^{2 n-1} \in \mathbb{C}^{2 n} \\
& \mathbb{H}^{n} \ni\left(x_{4 i}+x_{4 i+1} \mathfrak{i}+x_{4 i+2} \mathfrak{j}+x_{4 i+3} \mathfrak{k}\right)_{i=0}^{n-1} \longmapsto\left(x_{j}\right)_{j=0}^{4 n-1} \in \mathbb{R}^{4 n} .
\end{aligned}
$$

We also treat $\mathbb{H}^{n}$ as a hypercomplex manifold with the complex structures $I, J, K$ induced by the right multiplication by $\mathfrak{i}, \mathfrak{j}, \mathfrak{k}$, respectively (see [20] for details).

A function $f$ in a domain $\Omega \subset \mathbb{H}^{n}$ is called quaternionic plurisubharmonic, qpsh for short, if it is upper-semi-continuous and subharmonic when restricted to any slice 
of $\Omega$ by a right quaternionic line. The set of qpsh functions in $\Omega$ will be denoted by $\mathcal{Q P S H}(\Omega)$. These functions were introduced and studied in [1]. For a smooth function $f$ and the differential operators

$$
\begin{aligned}
\frac{\partial f}{\partial \overline{q_{k}}} & =\frac{\partial f}{\partial x_{4 k}}+\mathfrak{i} \frac{\partial f}{\partial x_{4 k+1}}+\mathfrak{j} \frac{\partial f}{\partial x_{4 k+2}}+\mathfrak{k} \frac{\partial f}{\partial x_{4 k+3}} \\
\frac{\partial f}{\partial q_{l}} & =\frac{\partial \bar{f}}{\partial \bar{q}_{l}}=\frac{\partial f}{\partial x_{4 l}}-\frac{\partial f}{\partial x_{4 l+1}} \mathfrak{i}-\frac{\partial f}{\partial x_{4 l+2}} \mathfrak{j}-\frac{\partial f}{\partial x_{4 l+3}} \mathfrak{k}
\end{aligned}
$$

being qpsh is equivalent to the fact that the matrix $\left[\frac{\partial^{2} f}{\partial \overline{q_{k}} \partial q_{l}}\right]_{k, l}$ is non-negative which means that $\overline{p_{k}} \frac{\partial^{2} f}{\partial \bar{q}_{k} \partial q_{l}} r_{l} \geq 0$ for all $p, r \in \mathbb{H}^{n}$. More details and references for the quaternionic linear algebra may be found in [1].

It is well known, cf. [1], that there is no determinant function defined on the set of all quaternionic matrices having all the properties of determinants of real or complex matrices. This causes the problem since it is tempting to define the quaternionic Monge-Ampère operator of $f$ as a determinant of $\left[\frac{\partial^{2} f}{\partial \bar{q}_{k} \partial q_{l}}\right]_{k, l}$. One may for instance consider the so-called Deieudonné or Study determinants but these are, among other disadvantages, always non-negative. Luckily, the matrix we obtain is hyperhermitian in the sense

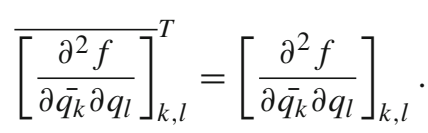

For such matrices Moore, cf. [17], has defined the notion of a determinant, we denote it by $\operatorname{det}_{M}$, which reflects enough properties of the determinant function. The original definition of quaternionic Monge-Ampère operator for smooth $f$ was just $\operatorname{det}_{M}\left[\frac{\partial^{2} f}{\partial \bar{q}_{k} \partial q_{l}}\right]_{k, l}$, cf. [1]. It was extended to continuous $f$ by a measure theoretic construction performed originally in the complex case in [6].

In [23] pluripotential theory was adopted to the quaternionic setting using methods from [6,7] and two formal operators $d_{0}, d_{1}$. As it was shown in [20] after a suitable choice in $[23]$ they coincide with $\partial$, the $(1,0)$ part with respect to the complex structure $I$ of an exterior differential $d$ in $\mathbb{H}^{n}$, and the differential operator $\partial_{J}$ defined by

$$
\partial_{J}=J^{-1} \circ \bar{\partial} \circ J
$$

This operators were studied in the context of the quaternionic Monge-Ampère equation for example in [5]. Because the operators $\partial, \partial_{J}$ are standard we use them in what follows.

Coming back to the content of [23], for a locally bounded $u \in \mathcal{Q P S H}(\Omega)$ a closed positive current $\partial \partial_{J} u$ was defined. This uses the notion of positivity, introduced by Alesker and Verbitsky in [4,5], for the forms $\alpha$ in $\mathbb{H}^{n}$ of a type $(2 k, 0)$ with respect to $I$ such that $J(\alpha)=\bar{\alpha}$. For us it is sufficient to know that $\sum_{i=0}^{n-1} d z_{2 i} \wedge d z_{2 i+1}$ (corresponding to $\sum d z_{i} \wedge d \overline{z_{i}}$ from the complex setting) and $\Omega_{n}=d z_{0} \wedge d z_{1} \wedge \cdots \wedge$ 
$d z_{2 n-2} \wedge d z_{2 n-1}$ are positive forms. The quaternionic Monge-Ampère operator for locally bounded functions is then defined by $\left(\partial \partial_{J} u\right)^{n}$ which is a Borel measure in $\Omega$. This definition actually gives a multiple of the operator defined by Alesker since, as checked for example in [4],

$$
\left(\partial \partial_{J} u\right)^{n}=\frac{n !}{4^{n}} \operatorname{det}_{M}\left[\frac{\partial^{2} u}{\partial_{\overline{q_{k}}} \partial_{q_{l}}}\right]_{k, l} \Omega_{n} .
$$

This also explains the convention to integrate $(2 n, 0)$ forms with respect to $I$ in $\mathbb{H}^{n}$, namely for $\alpha \in \Lambda_{I}^{2 n, 0}(\Omega)$ we define $\int_{\Omega} \alpha:=\int_{\Omega} f d \mathcal{L}^{4 n}$ if $\alpha=f \Omega_{n}$. The details concerning this approach can be found in [22-24].

For the rest of the paper, we denote by $\Omega$ a fixed quaternionic strictly pseudoconvex domain i.e. a $C^{2}-$ smoothly bounded domain such that there exists $\rho \in \mathcal{Q P S H}(U) \cap$ $C^{2}(U), \Omega \subset \subset U, \Omega=\{\rho<0\}, d \rho \neq 0$ on $\partial \Omega$ and $\left(\partial \partial_{J} \rho\right)^{n} \geq \Omega_{n}$ on $U$. We will also use the notion of quaternionic capacity, which we denote by cap, introduced in [24]. For a compact set, $K$ it is given by

$$
\operatorname{cap}(K, \Omega)=\sup \left\{\int_{K}\left(\partial \partial_{J} u\right)^{n} \mid u \in \mathcal{Q P} \mathcal{P H}(\Omega), 0 \leq u \leq 1\right\},
$$

while for a Borel set $E$

$$
\operatorname{cap}(E, \Omega)=\sup \{\operatorname{cap}(K) \mid K \text { is compact in } \Omega\},
$$

see [24] for details. Let $0 \leq \alpha \leq 1$, we denote by $\operatorname{Lip}_{\alpha}(\bar{\Omega})$ the space of $\alpha$-Hölder continues functions i.e. $f \in \operatorname{Lip}_{\alpha}(\bar{\Omega})$ if $f \in C^{0}(\bar{\Omega})$ and

$$
\|f\|_{\text {Lip }_{\alpha}(\bar{\Omega})}=\sup _{\bar{\Omega}} f+\sup _{p, q \in \bar{\Omega}, p \neq q} \frac{\|f(p)-f(q)\|}{\|p-q\|^{\alpha}}<+\infty .
$$

In the next two sections we perform many estimates. We use the notation $C(\cdot, \bullet, \ldots)$ for a constant depending only on the quantities $\cdot, \bullet, \ldots$ In particular $C$ 's depending on the same set of variables may vary from line to line.

\section{Stability of Solutions to the Quaternionic Monge-Ampère Equation}

In this section, we prove a version of the stability estimate for the quaternionic MongeAmpère equation. In [20] it was proven that the uniform norm of the difference of solutions is under control of the uniform norm of the difference of boundary data and the $L^{q}$ norm of the difference of Monge-Ampère densities when they belong to $L^{q}$ for $q>2$, cf. Proposition 4 in [20]. Our goal here is to prove that the uniform norm of the difference of solutions is under control of the $L^{p}$ norm of that difference for appropriate $p$, cf. Theorem 1 below.

For the purposes of this section we set the following convention. Whenever $u \in$ $\mathcal{Q P S H}(\Omega)$ is locally bounded then writing $\left\|\left(\partial \partial_{J} u\right)^{n}\right\|_{p}$ automatically implies that 
we assume the Borel measure $\left(\partial \partial_{J} u\right)^{n}$ has a density with respect to the Lebesgue measure $\mathcal{L}^{4 n}$ and this density is in $L^{p}(\Omega)$. We will need the following comparison of quaternionic capacity and Lebesgue measure. Its proof uses first author's $C^{0}$ estimate for the complex Monge-Ampère equation which in turn uses the comparison of the complex capacity and the Lebesgue measure, cf. $[15,16]$.

Lemma 1 ([20]) For a fixed $p \in(1,2)$ there exists a constant $C(p, R)$ such that for any $\Omega \subset B(0, R)$ and any Borel set $E \subset \Omega$

$$
\mathcal{L}^{4 n}(E) \leq C(p, R) \operatorname{cap}^{p}(E, \Omega) .
$$

Remark In the given reference this lemma is shown for compact sets but then the inequality follows easily for any Borel set with the same constant.

Another technical result which we need is the lemma below. It was used implicitly back in [14], since then it turned out to be very useful while performing pluripotential estimates. Its form below is a combination of Lemma 1.5 in [11] and Lemma 2.4 in [9]. In [19] it was attributed to De Giorgi.

Lemma 2 (De Giorgi) Suppose that a non-increasing, right continuous function $f$ : $[0, \infty) \rightarrow[0, \infty]$ such that $\lim _{x \rightarrow \infty} f(x)=0$ satisfies

$$
\text { (*) } t f(t+s) \leq B f(s)^{1+\alpha}
$$

for any $s \geq 0, t \in[0,1]$ and some $\alpha, B>0$. There exists $s_{\infty}$, depending on $\alpha, B$ and $s_{0}$ such that $f\left(s_{0}\right) \leq \frac{1}{2 B}$, satisfying $f(s)=0$ for any $s \geq s_{\infty}$. In fact one can choose $s_{\infty}$ to be equal $s_{0}+\frac{2 B f\left(s_{0}\right)^{\alpha}}{1-2^{-\alpha}}$. If in addition $(*)$ holds for all $s, t \geq 0$ then one can take even $s_{\infty}=\frac{2 B f(0)^{\alpha}}{1-2^{-\alpha}}$.

Now, we show the main technical fact needed for the proof of Theorem 1 . The reasoning we perform in the rest of the section is based on the one presented in [11].

Proposition 1 Fix $c_{0}>0$ and $p>2$. Let $u, v \in \mathcal{Q P S H}(\Omega) \cap L_{l o c}^{\infty}(\Omega)$ be such that

$$
\begin{aligned}
& \liminf _{q \rightarrow q_{0}}(u-v)(q) \geq 0 \text { for any } q_{0} \in \partial \Omega, \\
& \left\|\left(\partial \partial_{J} u\right)^{n}\right\|_{L^{p}(\Omega)} \leq c_{0} .
\end{aligned}
$$

For $0<\alpha<\frac{p-2}{n p}$ there exists a constant $C\left(c_{0}, \alpha\right.$, diam $\left.(\Omega)\right)$, depending on $c_{0}, \alpha$ and the diameter of $\Omega$, such that for any $\epsilon>0$

$$
\sup (v-u) \leq \epsilon+C\left(c_{0}, \alpha, \operatorname{diam}(\Omega)\right)(\operatorname{cap}(\{u-v<-\epsilon\}, \Omega))^{\alpha} .
$$

Proof Define

$$
U_{\epsilon}(s)=\{u-v<-\epsilon-s\} \text { and } b_{\epsilon}(s)=\left(\operatorname{cap}\left(U_{\epsilon}(s), \Omega\right)\right)^{\frac{1}{n}}
$$

for $s \geq 0$ and $\epsilon>0$. Firstly, note that for all $t, s \geq 0, \epsilon>0$ and $w \in \mathcal{Q P \mathcal { S H }}(\Omega)$ such that $0 \leq w \leq 1$ one obtains from inclusions of sets, super-additivity and the comparison principle, cf. Theorem 1.2 in [24], 


$$
\begin{aligned}
t^{n} & \int_{U_{\epsilon}(s+t)}\left(\partial \partial_{J} w\right)^{n} \\
& =\int_{U_{\epsilon}(s+t)}\left(\partial \partial_{J}(t w-t-s-\epsilon)\right)^{n}=\int_{\{u<v-s-t-\epsilon\}}\left(\partial \partial_{J}(t w-t-s-\epsilon)\right)^{n} \\
& \leq \int_{\{u<v-s+t w-t-\epsilon\}}\left(\partial \partial_{J}(t w-t-s-\epsilon)\right)^{n} \\
& \leq \int_{\{u<v-s+t w-t-\epsilon\}}\left(\partial \partial_{J}(v+t w-t-s-\epsilon)\right)^{n} \\
& \leq \int_{\{u<v-s+t w-t-\epsilon\}}\left(\partial \partial_{J} u\right)^{n} \leq \int_{\{u<v-s-\epsilon\}}\left(\partial \partial_{J} u\right)^{n}=\int_{U_{\epsilon}(s)}\left(\partial \partial_{J} u\right)^{n} .
\end{aligned}
$$

From the Hölder inequality we obtain

$$
\begin{aligned}
\int_{U_{\epsilon}(s)}\left(\partial_{J} u\right)^{n} & \leq\left\|\left(\partial_{J} u\right)^{n}\right\|_{L^{p}(\Omega)}\left(\mathcal{L}^{4 n}\left(U_{\epsilon}(s)\right)\right)^{\frac{1}{p^{\prime}}} \\
& \leq C\left(q^{\prime}, \operatorname{diam}(\Omega)\right) c_{0}\left(\operatorname{cap}\left(U_{\epsilon}(s), \Omega\right)\right)^{\frac{q^{\prime}}{p^{\prime}}} \\
& =C\left(c_{0}, \alpha, \operatorname{diam}(\Omega)\right)\left(b_{\epsilon}(s)\right)^{n(1+n \alpha)}
\end{aligned}
$$

where $q^{\prime} \in(1,2)$ depends only on $p^{\prime}$ which is the conjugate of $p$ and we choose it so that $\frac{q^{\prime}}{p^{\prime}}=1+n \alpha$. Since $1+n \alpha<\frac{2}{p^{\prime}}$ this is always possible. Taking the supremum over all $w$ and $n$ 'th root of both sides gives

$$
t b_{\epsilon}(s+t) \leq C\left(c_{0}, \alpha, \operatorname{diam}(\Omega)\right)\left(b_{\epsilon}(s)\right)^{1+n \alpha}
$$

for any $s, t \geq 0$ and $\epsilon>0$. One easily checks, as in [19], that the function $b_{\epsilon}$ satisfies all the assumptions of Lemma 2. This gives

$$
\operatorname{cap}\left(\left\{u-v<-\epsilon-\frac{2 C\left(c_{0}, \alpha, \operatorname{diam}(\Omega)\right)}{1-2^{-n \alpha}} b_{\epsilon}(0)^{n \alpha}\right\}, \Omega\right)=0 .
$$

From the comparison of volume and capacity (see Lemma 1) it follows that $v-u \leq$ $\epsilon+C\left(c_{0}, \alpha, \operatorname{diam}(\Omega)\right) b_{\epsilon}(0)^{n \alpha}$ almost everywhere in $\Omega$. Since $u$ and $v$ are subharmonic we obtain that this holds in $\Omega$, i.e.

$$
\sup _{\Omega}(v-u) \leq \epsilon+C\left(c_{0}, \alpha, \operatorname{diam}(\Omega)\right)\left(\operatorname{cap}\left(U_{\epsilon}(0), \Omega\right)\right)^{\alpha} .
$$

We are ready to prove the announced stability of weak solutions.

Theorem 1 Fix $c_{0}>0$ and $p>2$. Let $u, v \in \mathcal{Q P S H}(\Omega) \cap L_{\text {loc }}^{\infty}(\Omega)$ be such that

$$
\liminf _{q \rightarrow q_{0}}(u-v)(q) \geq 0 \text { for any } q_{0} \in \partial \Omega,
$$




$$
\left\|\left(\partial \partial_{J} u\right)^{n}\right\|_{L^{p}(\Omega)} \leq c_{0} .
$$

For any $r \geq 1$ and $0<\gamma<\frac{r}{r+n p^{\prime}+\frac{p^{\prime} n p}{p-2}}:=\gamma_{r}$ there exists a constant $C\left(c_{0}, \gamma\right.$, diam $\left.(\Omega)\right)$, depending only on $c_{0}, \gamma$ and the diameter of $\Omega$, such that

$$
\sup _{\Omega}(v-u) \leq C\left(c_{0}, \gamma, \operatorname{diam}(\Omega)\right)\left\|(v-u)_{+}\right\|_{L^{r}(\Omega)}^{\gamma} \cdot
$$

Proof First of all, we may assume that $\left\|(v-u)_{+}\right\|_{L^{r}(\Omega)} \neq 0$ because otherwise the inequality holds with any constant $C\left(c_{0}, \gamma, \operatorname{diam}(\Omega)\right)$. Arguing as in the beginning of the proof of Proposition 1 we obtain for any $\epsilon>0$

$$
\operatorname{cap}(\{u-v<-2 \epsilon\}, \Omega) \leq \epsilon^{-n} \int_{\{u-v<-\epsilon\}}\left(\partial \partial_{J} u\right)^{n} .
$$

Since on the set $\{u-v<-\epsilon\}$ the function $\left(\left(\frac{v-u}{\epsilon}\right)_{+}\right)^{\frac{r}{p^{\prime}}}$ is bigger than 1 , due to Hölder's inequality we may further estimate

$$
\begin{aligned}
& \epsilon^{-n} \int_{\{u-v<-\epsilon\}}\left(\partial_{J} u\right)^{n} \\
& \quad \leq \epsilon^{-n-\frac{r}{p^{\prime}}} \int_{\Omega}(v-u)_{+}^{\frac{r}{p^{\prime}}}\left(\partial \partial_{J} u\right)^{n} \\
& \quad \leq \epsilon^{-n-\frac{r}{p^{\prime}}}\left\|\left(\partial \partial_{J} u\right)^{n}\right\|_{L^{p}(\Omega)}\left(\left(\int_{\Omega}\left((v-u)_{+}\right)^{r}\right)^{\frac{1}{r}}\right)^{\frac{r}{p^{\prime}}} \\
& \leq \epsilon^{-n-\frac{r}{p^{\prime}}}\left\|\left(\partial \partial_{J} u\right)^{n}\right\|_{L^{p}(\Omega)}\left\|(v-u)_{+}\right\|_{L^{r}(\Omega)}^{\frac{r}{p^{\prime}}} \\
& \leq \epsilon^{-n-\frac{r}{p^{\prime}}} c_{0}\left\|(v-u)_{+}\right\|_{L^{r}(\Omega)}^{\frac{r}{p^{\prime}}} \cdot
\end{aligned}
$$

Applying Proposition 1 we get

$$
\begin{aligned}
\sup _{\Omega}(v-u) & \leq 2 \epsilon+C\left(c_{0}, \alpha, \operatorname{diam}(\Omega)\right)(\operatorname{cap}(\{u-v<-2 \epsilon\}, \Omega))^{\alpha} \\
& \leq 2 \epsilon+C\left(c_{0}, \alpha, \operatorname{diam}(\Omega)\right) \epsilon^{-\alpha n-\frac{r \alpha}{p^{\prime}}} c_{0}^{\alpha}\left\|(v-u)_{+}\right\|_{L^{r}(\Omega)}^{\frac{r \alpha}{p^{\prime}}}
\end{aligned}
$$

for any $0<\alpha<\frac{p-2}{n p}$. Putting $\epsilon=\left\|(v-u)_{+}\right\|_{L^{r}(\Omega)}^{\gamma}$ gives

$$
\sup _{\Omega}(v-u) \leq 2\left\|(v-u)_{+}\right\|_{L^{r}(\Omega)}^{\gamma}+C\left(c_{0}, \alpha, \operatorname{diam}(\Omega)\right) c_{0}^{\alpha}\left\|(v-u)_{+}\right\|_{L^{r}(\Omega)}^{\gamma \alpha\left(-n-\frac{r}{p^{\prime}}\right)+\frac{r \alpha}{p^{\prime}}} .
$$


Choosing $\alpha$ such that $\gamma=\frac{r}{r+n p^{\prime}+\frac{p^{\prime}}{\alpha}}$ (which is always possible since when $\alpha$ varies in $\left(0, \frac{p-2}{n p}\right)$ the quantity $\frac{r}{r+n p^{\prime}+\frac{p^{\prime}}{\alpha}}$ varies in $\left.\left(0, \gamma_{r}\right)\right)$ results in

$$
\sup _{\Omega}(v-u) \leq C\left(c_{0}, \gamma, \operatorname{diam}(\Omega)\right)\left\|(v-u)_{+}\right\|_{L^{r}(\Omega)}^{\gamma}
$$

because $\gamma \alpha\left(-n-\frac{r}{p^{\prime}}\right)+\frac{r \alpha}{p^{\prime}}=\gamma\left(-\alpha n-\frac{\alpha r}{p^{\prime}}+\frac{r \alpha}{\gamma p^{\prime}}\right)=\gamma\left(-\alpha n-\frac{\alpha r}{p^{\prime}}+\frac{\alpha}{p^{\prime}}(r+\right.$ $\left.\left.n p^{\prime}+\frac{p^{\prime}}{\alpha}\right)\right)=\gamma$.

\section{Hölder Continuity of Weak Solutions}

In this section, we proceed to proving regularity of solutions to the Dirichlet problem for the quaternionic Monge-Ampère equation under the conditions on the boundary data and the density. This is the content of Theorem 3. For that goal we first consider a more general situation in Theorem 2 below and then check that under the assumptions of Theorem 3 one can apply Theorem 2 .

Theorem 2 Let $\Omega \subset \mathbb{H}^{n}$ be a quaternionic strictly pseudoconvex domain. Suppose $p>2, f \in L^{p}(\Omega)$ is a non-negative function, $\phi \in C(\partial \Omega)$ and $u$ is the solution to the Dirichlet problem

$$
\left\{\begin{array}{l}
u \in \mathcal{Q P} \mathcal{S H}(\Omega) \cap C(\bar{\Omega}) \\
\left(\partial_{J} u\right)^{n}=f \Omega_{n} \\
u_{\mid \partial \Omega}=\phi
\end{array}\right.
$$

such that $\Delta u(\Omega)$ is finite. If there exists $0<v<1$ and $b \in \operatorname{Lip}_{v}(\bar{\Omega})$ such that $b \leq u$ in $\Omega$ and $b=\phi$ on $\partial \Omega$ then $u \in \operatorname{Lip}_{\alpha}(\bar{\Omega})$ for any $0 \leq \alpha<\min \left\{v, 2 \gamma_{1}\right\}$.

Remark Let us just emphasize that in the whole section we denote by $\Delta u$, for a locally bounded $u$, the distributional Laplacian. Since qpsh functions are in particular subharmonic for them $\Delta u$ is a positive distribution thus a measure and so it makes sense to write $\Delta u(\Omega)$.

We introduce the notation needed for the proof of this theorem. This approach is similar to the one presented in [18]. For a fixed number $\delta>0$ and a subharmonic $u$ we consider

$$
\begin{aligned}
\Omega_{\delta} & =\{q \in \Omega \mid \operatorname{dist}(q, \Omega)>\delta\} \\
u_{\delta}(q) & =\sup _{\|p\| \leq \delta} u(q+p), \text { for } q \in \Omega_{\delta} \\
\hat{u_{\delta}}(q) & =\frac{1}{\mathcal{L}^{4 n}(B(0,1)) \delta^{4 n}} \int_{\|q-p\| \leq \delta} u(p) d \mathcal{L}^{4 n}(p), \text { for } q \in \Omega_{\delta} .
\end{aligned}
$$


The lemma below is a composition of Lemmas 4.2 and 4.3 in [11]. As was noted by Nguyen in [18] proofs given originally in [11] for plurisubharmonic functions used only subharmonicity.

Lemma 3 Let $u$ be a subharmonic function in $\Omega$. For a fixed $0<\alpha<1$ the following are equivalent

(i) there exists $\delta_{0}, A>0$ such that for any $0<\delta \leq \delta_{0}$

$$
u_{\delta}-u \leq A \delta^{\alpha} \text { in } \Omega_{\delta}
$$

(ii) there exists $\delta_{1}, B>0$ such that for any $0<\delta<\delta_{1}$

$$
\hat{u_{\delta}}-u \leq A \delta^{\alpha} \text { in } \Omega_{\delta} \text {. }
$$

Moreover, there exists a constant $c$, depending only on the dimension $n$, such that for all $\delta>0$ sufficiently small

$$
\int_{\Omega_{\delta}}\left(\hat{u_{\delta}}(q)-u(q)\right) d \mathcal{L}^{4 n}(q) \leq c \Delta u(\Omega) \delta^{2} .
$$

Lemma 4 Let $u$ and $b$ be as in Theorem 2. There exists a constant $c_{n}$ such that for all $0<\delta$ and $q \in \partial \Omega_{\delta}$

$$
u_{\delta}(q) \leq u(q)+c_{n} \delta^{\nu}
$$

Proof Denote by $h$ the harmonic extension of $b_{\mid \partial \Omega}$ to $\Omega$. By the Proposition 2.4 in [18] we know that $h \in \operatorname{Lip}_{v}(\bar{\Omega})$. Fix $q \in \partial \Omega_{\delta}$ and take $p, p_{0} \in \mathbb{H}^{n}$ such that $\|p\|=\left\|p_{0}\right\|=\delta, u_{\delta}(q)=u(p+q)$ and $q+p_{0} \in \partial \Omega$. Since $b \leq u \leq h$ in $\bar{\Omega}$, with equalities on $\partial \Omega$, we obtain the following string of inequalities

$$
\begin{aligned}
u_{\delta}(q)-u(q)= & u(p+q)-u(q) \leq h(p+q)-u(q) \leq h(p+q)-b(q) \\
= & h(p+q)-h(q)+h(q)-b(q) \leq\|h\|_{L_{i p}(\bar{\Omega})} \delta^{v}+h(q) \\
& -h\left(q+p_{0}\right)+b\left(q+p_{0}\right)-b(q) \leq\left(2\|h\|_{L_{i p}(\bar{\Omega})}\right. \\
& \left.+\|b\|_{L_{p_{v}}(\bar{\Omega})}\right) \delta^{v} .
\end{aligned}
$$

Proof of Theorem 2 Fix $0<\gamma<\gamma_{1}$ and consider the function

$$
\tilde{u}_{\delta}(q)=\left\{\begin{array}{l}
\max \left\{\hat{u}_{\delta}(q), u(q)+c_{n} \delta^{\nu}\right\}, \text { for } q \in \Omega_{\delta} \\
u(q)+c_{n} \delta^{\nu}, \text { for } q \in \bar{\Omega} \backslash \Omega_{\delta},
\end{array}\right.
$$

which by Lemma 4 , is qpsh in $\Omega$, as $\hat{u}_{\delta} \leq u_{\delta}$ (cf. Proposition 2.1(4) in [22]); and continuous in $\bar{\Omega}$. Applying Theorem 1 for $u+c_{n} \delta^{v}, \tilde{u}_{\delta}$ and $r=1$ we obtain

$$
\sup _{\Omega}\left(\tilde{u}_{\delta}-u-c_{n} \delta^{\nu}\right) \leq C\left(\|f\|_{p}, \gamma, \operatorname{diam}(\Omega)\right)\left\|\left(\tilde{u}_{\delta}-u-c_{n} \delta^{\nu}\right)_{+}\right\|_{L^{1}(\Omega)}^{\gamma} .
$$


From the construction of $\tilde{u}_{\delta}$ it follows that the last inequality is equivalent to

$$
\sup _{\Omega_{\delta}}\left(\hat{u}_{\delta}-u-c_{n} \delta^{\nu}\right) \leq C\left(\|f\|_{p}, \gamma, \operatorname{diam}(\Omega)\right)\left\|\left(\hat{u}_{\delta}-u-c_{n} \delta^{\nu}\right)_{+}\right\|_{L^{1}\left(\Omega_{\delta}\right)}^{\gamma}
$$

Estimating further, by Lemma 3 and the trivial estimate $\left(\hat{u}_{\delta}-u-c_{n} \delta^{v}\right)_{+} \leq \hat{u}_{\delta}-u$, we obtain for sufficiently small $\delta>0$

$$
\sup _{\Omega_{\delta}}\left(\hat{u}_{\delta}-u-c_{n} \delta^{\nu}\right) \leq c^{\gamma} C\left(\|f\|_{p}, \gamma, \operatorname{diam}(\Omega)\right)(\Delta u(\Omega))^{\gamma} \delta^{2 \gamma}
$$

This results in

$$
\sup _{\Omega_{\delta}}\left(\hat{u}_{\delta}-u\right) \leq C\left(\|f\|_{p}, \gamma, \operatorname{diam}(\Omega), \Delta u(\Omega), c, c_{n}\right) \delta^{\min \{v, 2 \gamma\}}
$$

for all $\delta$ sufficiently small. Since the constant $C$ is independent of $\delta$ we obtain, again due to Lemma 3, that $u \in \operatorname{Lip}_{\min \{v, 2 \gamma\}}(\bar{\Omega})$. Since $\gamma$ was arbitrary in $\left(0, \gamma_{1}\right)$ this gives our claim.

Theorem 3 Let $\Omega \subset \mathbb{H}^{n}$ be a quaternionic strictly pseudoconvex domain. Suppose $p>2, f \in L^{p}(\Omega)$ is a non-negative function bounded in a neighborhood of $\partial \Omega$ and $\phi \in C^{1,1}(\partial \Omega)$. Then the Dirichlet problem

$$
\left\{\begin{array}{l}
u \in \mathcal{Q P \mathcal { S H }}(\Omega) \cap C(\bar{\Omega}) \\
\left(\partial_{J} u\right)^{n}=f \Omega_{n} \\
u_{\mid \partial \Omega}=\phi
\end{array}\right.
$$

is solvable and the unique solution is in $\operatorname{Lip}_{\alpha}(\bar{\Omega})$ for any $0 \leq \alpha \leq 2 \gamma_{1}$.

Proof The continuous solution $u$ exists and is unique as was shown in [20]. We need to check the assumptions of Theorem 2. For that goal, we construct a function $b$ as in Theorem 2 having in addition the properties that it is subharmonic and the Laplacian of it has finite total mass. This will of course imply that the Laplacian of $u$ has finite total mass since $b$ and $u$, both subharmonic, will agree on $\partial \Omega$ and $b \leq u$ in $\Omega$. Take $h$ to be the solution to the Dirichlet problem

$$
\left\{\begin{array}{l}
h \in \mathcal{Q P \mathcal { S H }}(\Omega) \cap C(\bar{\Omega}) \\
\left(\partial_{J} h\right)^{n}=0 \\
h_{\mid \partial \Omega}=\phi
\end{array}\right.
$$

By the comparison principle, cf. Theorem 1.2 in [24], it is above any $v \in \mathcal{Q P \mathcal { H }}(\Omega) \cap$ $C(\bar{\Omega})$ such that $v_{\mid \partial \Omega}=\phi$.

We will show that $h$ is Lipschitz in $\bar{\Omega}$ and its Laplacian has finite total mass in $\Omega$. Suppose $U$ is a neighborhood of $\bar{\Omega}$ such that $\phi$ is extendable to a function $\hat{\phi} \in C^{1,1}(\bar{U})$. 
That is always possible due to Lemmas 6.37 and 6.38 in [10]. Consider a defining function $\rho$ of $\Omega$ in the neighborhood of $\bar{U}$ i.e.

$$
\begin{aligned}
& \rho \in \mathcal{Q P \mathcal { S H }}(\bar{U}) \cap C^{2}(\bar{U}), \\
& \Omega=\{\rho<0\}, \rho_{\mid \partial \Omega}=0, d \rho \neq 0 \text { on } \partial \Omega, \\
& \left(\partial \partial_{J} \rho\right)^{n} \geq \Omega_{n} \text { on } \bar{\Omega} .
\end{aligned}
$$

We take $A$ big enough such that $A \rho+\hat{\phi}$ and $A \rho-\hat{\phi}$ are in $\mathcal{Q P} \mathcal{S H}(U)$. Note that $A \rho+\hat{\phi} \leq h$ in $\Omega$ from the definition of $h$. This shows that the Laplacian of $h$ has a finite total mass in $\Omega$ since $A \rho+\hat{\phi}$ has this property and that the function $\hat{h}$ defined by

$$
\hat{h}(q)=\left\{\begin{array}{l}
h(q), \text { for } q \in \Omega \\
A \rho(q)+\hat{\phi}(q), \text { for } q \in \bar{U} \backslash \Omega
\end{array}\right.
$$

belongs to $\mathcal{Q P S H}(U) \cap C^{0}(\bar{U})$, cf. Proposition 2.1(4) in [22]. Take $\epsilon>0$ such that $\Omega^{(\epsilon)}=\left\{q \in \mathbb{H}^{n} \mid \operatorname{dist}(q, \bar{\Omega})<\epsilon\right\} \subset \subset U$. For any $p \in \partial \Omega$ and $q$ such that $\|q\|<\epsilon$ we have

$$
\begin{aligned}
\hat{h}(p+q) \leq & \hat{\phi}(p)+\max \left\{\|A \rho \pm \hat{\phi}\|_{C^{1}(\bar{U})}\right\}\|q\|=\phi(p) \\
& +\max \left\{\|A \rho \pm \hat{\phi}\|_{C^{1}(\bar{U})}\right\}\|q\|
\end{aligned}
$$

due to the mean value theorem and because $\hat{h}=h \leq \hat{\phi}-A \rho$ in $\Omega$ (as the subharmonic function $h-\hat{\phi}+A \rho$ attains its maximum (equal zero) on the boundary of $\Omega$ ). Thus for $C=\max \left\{\|A \rho \pm \hat{\phi}\|_{C^{1}(\bar{U})}\right\}$, every $p \in \partial \Omega$ and $\|q\|<\epsilon$ we have

$$
\hat{h}(p+q)-C\|q\| \leq \phi(p)
$$

From the properties of $h$ it means that for any $\|q\|<\epsilon$

$$
\hat{h}(r+q)-C\|q\| \leq h(r) \text { for all } r \in \bar{\Omega} .
$$

For $r \in \bar{\Omega}$ and $\|q\| \leq \epsilon$ such that $r+q \in \bar{\Omega}$ we thus obtain

$$
\begin{aligned}
\hat{h}(r+q)-h(r) & =h(r+q)-h(r) \leq C\|q\|, \\
\hat{h}(r+q-q)-h(r+q) & =h(r)-h(r+q) \leq C\|-q\|,
\end{aligned}
$$

what results in

$$
\|h(r+q)-h(r)\| \leq C\|q\| \text { for } r \in \bar{\Omega} \text { and }\|q\|<\epsilon \text { such that } r+q \in \bar{\Omega}
$$

This shows that $h$ is locally Lipschitz continuous in $\bar{\Omega}$ and consequently Lipschitz continuous. 
By the assumptions for some $M>0$ we have $f \leq M$ away from a compact $K \subset \Omega$. Let $u$ be the continuous solution to our Dirichlet problem. Take $B$ big enough for the function $B \rho+h$ to be below $u$ in a neighborhood of $K$ and such that $B^{n}>M$. Then by supper-additivity

$$
\left(\partial \partial_{J}(B \rho+h)\right)^{n} \geq\left(\partial \partial_{J} B \rho\right)^{n} \geq f \Omega_{n}
$$

at least in $\Omega \backslash K$. The comparison principle, cf. [24], implies that $B \rho+h \leq u$ in $\Omega \backslash K$ since the inequality holds on the boundary of this set.

We define $b=B \rho+h$. It is Lipschitz continuous and its Laplacian has finite total mass in $\Omega$ since $h$ has these properties.

Acknowledgements The authors Sławomir Kołodziej and Marcin Sroka were partially supported by the National Science Center, Poland; Grant No. 2017/27/B/ST1/01145.

Open Access This article is licensed under a Creative Commons Attribution 4.0 International License, which permits use, sharing, adaptation, distribution and reproduction in any medium or format, as long as you give appropriate credit to the original author(s) and the source, provide a link to the Creative Commons licence, and indicate if changes were made. The images or other third party material in this article are included in the article's Creative Commons licence, unless indicated otherwise in a credit line to the material. If material is not included in the article's Creative Commons licence and your intended use is not permitted by statutory regulation or exceeds the permitted use, you will need to obtain permission directly from the copyright holder. To view a copy of this licence, visit http://creativecommons.org/licenses/by/4.0/.

\section{References}

1. Alesker, S.: Non-commutative linear algebra and plurisubharmonic functions of quaternionic variables. Bull. Sci. Math. 127(1), 1-35 (2003)

2. Alesker, S.: Quaternionic Monge-Ampère equations. J. Geom. Anal. 13(2), 205-238 (2003)

3. Alesker, S.: Pluripotential theory on quaternionic manifolds. J. Geom. Phys. 62(5), 1189-1206 (2012)

4. Alesker, S., Verbitsky, M.: Plurisubharmonic functions on hypercomplex manifolds and HKTgeometry. J. Glob. Anal. 16, 375-399 (2006)

5. Alesker, S., Verbitsky, M.: Quaternionic Monge-Ampère equations and Calabi problem for HKTmanifolds. Israel J. Math. 176, 109-138 (2010)

6. Bedford, E., Taylor, B.A.: The Dirichlet problem for a complex Monge-Ampère equation. Invent. Math. 37(1), 1-44 (1976)

7. Bedford, E., Taylor, B.A.: A new capacity for plurisubharmonic functions. Acta Math. 149, 1-40 (1982)

8. Caffarelli, L., Kohn, J.J., Nirenberg, L., Spruck, J.: The dirichlet problem for nonlinear second-order elliptic equations. II. Complex Monge-Ampère, and uniformly elliptic equations. Commun. Pure Appl. Math. 38, 209-252 (1985)

9. Eyssidieux, P., Guedj, V., Zeriahi, A.: Singular Kähler-Einstein metrics. J. Am. Math. Soc. 22, 607-639 (2009)

10. Gilbarg, D., Trudinger, N.: Elliptic Partial Differential Equations of Second Order. Springer, Berlin (2001)

11. Guedj, V., Kołodziej, S., Zeriahi, A.: Hölder continuous solutions to Monge-Ampère equations. Bull. Lond. Math. Soc. 40, 1070-1080 (2008)

12. Harvey, F.R., Lawson Jr., B.L.: Dirichlet duality and the nonlinear dirichlet problem. Commun. Pure Appl. Math. 62, 396-443 (2009)

13. Harvey, F.R., Lawson Jr., B.L.: An introduction to potential theory in calibrated geometry. Am. J. Math. 131(4), 893-944 (2009)

14. Kołodziej, S.: Some sufficient conditions for solvability of the Dirichlet problem for the complex Monge-Ampère operator. Ann. Polon. Math. 65, 11-21 (1996) 
15. Kołodziej, S.: The complex Monge-Ampère equation. Acta Math. 180, 69-117 (1998)

16. Kołodziej, S.: The complex Monge-Ampère equation and pluripotential theory. Mem. Am. Math. Soc. 178, 840 (2005)

17. Moore, E.: On the determinant of an hermitian matrix of quaternionic elements. Bull. Am. Math. Soc. 28, 161-162 (1922)

18. Nguyen, N.C.: Hölder continuous solutions to complex Hessian equations. Potential Anal. 41(3), 887902 (2014)

19. Phong, D.H., Song, J., Sturm, J.: Complex Monge-Ampère equations in surveys in differential. Geometry 17, 327-411 (2012)

20. Sroka, M.: Weak solutions to the quaternionic Monge-Ampère equation, to appear in Analysis \& PDE

21. Verbitsky, M.: HyperKähler manifolds with torsion, supersymmetry and Hodge theory. Asian J. Math. 6(4), 679-712 (2002)

22. Wan, D., Kang, Q.: Pluripotential theory for quaternionic plurisubharmonic functions. Mich. Math. J. 66, 3-20 (2017)

23. Wan, D., Wang, W.: On the quaternionic Monge-Ampère operator, closed positive currents and LelongJensen type formula on the quaternionic space. Bull. Sci. Math. 141, 267-311 (2017)

24. Wan, D., Zhang, W.: Quasicontinuity and maximality of quaternionic plurisubharmonic functions. J. Math. Anal. Appl. 424, 86-103 (2015)

25. Zhu, J.: Dirichlet problem of quaternionic Monge-Ampère equations. Israel J. Math. 214(2), 597-619 (2017)

Publisher's Note Springer Nature remains neutral with regard to jurisdictional claims in published maps and institutional affiliations. 